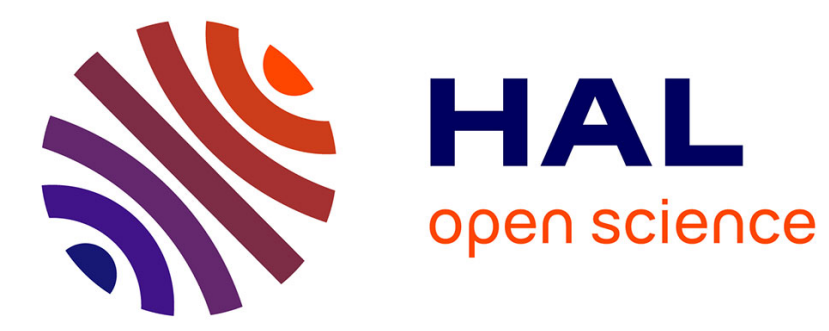

\title{
Discrete linear objects in dimension n: the standard model
}

Eric Andres

\section{To cite this version:}

Eric Andres. Discrete linear objects in dimension n: the standard model. Graphical Models, 2003, 65 (1-3), pp.92 - 111. 10.1016/S1524-0703(03)00004-3 . hal-00354729

\section{HAL Id: hal-00354729 \\ https://hal.science/hal-00354729}

Submitted on 21 Jan 2009

HAL is a multi-disciplinary open access archive for the deposit and dissemination of scientific research documents, whether they are published or not. The documents may come from teaching and research institutions in France or abroad, or from public or private research centers.
L'archive ouverte pluridisciplinaire HAL, est destinée au dépôt et à la diffusion de documents scientifiques de niveau recherche, publiés ou non, émanant des établissements d'enseignement et de recherche français ou étrangers, des laboratoires publics ou privés. 


\title{
Discrete Linear Objects in Dimension n: the Standard Model
}

\author{
Eric ANDRES ${ }^{a}$ \\ a IRCOM-SIC, SP2MI, BP 30179, F-86962 Futuroscope Cedex, France
}

\begin{abstract}
A new analytical description model, called the standard model, for the discretization of Euclidean linear objects (point, $m$-flat, $m$-simplex) in dimension $n$ is proposed. The objects are defined analytically by inequalities. This allows a global definition independent of the number of discrete points. A method is provided to compute the analytical description for a given linear object. A discrete standard model has many properties in common with the supercover model from which it derives. However, contrary to supercover objects, a standard object does not have bubbles. A standard object is $(n-1)$-connected, tunnel-free and bubble-free. The standard model is geometrically consistent. The standard model is well suited for modelling applications.
\end{abstract}

Key words: discrete geometry, digitization, dimension n, simplexe, m-flat

Email address: andres@sic.univ-poitiers.fr (Eric ANDRES).

Preprint submitted to Graphical Models

4 November 2002 


\section{Introduction}

When working in discrete geometry, aside from considering an object simply as a set of discrete points, the problem of defining discrete geometrical objects arises. A discrete 2D line segment can be defined as 8-connected, 4-connected or even disconnected as a dotted line. There is not a unique way of defining a discrete object or of digitizing a Euclidean object. This problem has been around for forty years and many different discrete object definitions have been proposed. One can say that authors have followed three main approaches to define discrete geometrical objects: an algorithmic approach, a topological approach and a more recent analytical approach followed in this paper. In the algorithmic approach $[1,10,13,16,21-24,34]$ a discrete object is the result of a generation algorithm. Historically, the first approach that has been used, it has shown a number of limitations. It is often difficult to control the properties of the so defined discrete objects. For instance, the discrete objects might not be geometrically consistent : the edge of a 3D triangle is typically not necessary a 3D line segment or the $3 \mathrm{D}$ triangle is not a piece of $3 \mathrm{D}$ plane $[21,22]$. It is also difficult to propose generation algorithms for discrete objects in dimension higher than three. Except for n-dimensional lines [34], to the best authors knowledge, no discrete object, in dimensions higher than three, has been algorithmically defined. In the topological approach, a discrete object is typically defined as a class of objects verifying local properties, often topological in nature [18-20,25,28]. While it is, by definition, easier to obtain the desired properties, it is difficult to be sure with such an approach, that the class of objects defined by a given set of properties is not larger than what is initially expected. A third, more recent approach, defines a discrete object 
by a global analytical definition [2-5,7,9,17,18,25,30,32]. This approach has many advantages such as providing a compact definition (independent on the number of points forming the discrete object), a global control of the discrete object. It has also an advantage that is not immediatly visible when one is not familiar with this approach. It allows a good control of the local topological properties of the object. The many links with mathematical morphology are also an interesting property of some analytically defined models such as the supercover model $[7,19,26,29,31,33]$. One of the main advantages is that it is relatively easy to define discrete objects in an arbitrary dimension $[3,4,7,30,32]$. The standard model introduced in the following pages is analytically defined.

A new analytical description model for all linear objects in dimension $n$ (discrete points, $m$-flats and geometrical simplices) is presented in this paper. The analytical model is called the standard model. The names derives from the name given by J. Françon [18] to $(n-1)$-connected analytical discrete 3D planes (see also [4] for general details on discrete analytical hyperplanes). To the best authors knowledge, it is the first time that a discrete model is proposed that defines a large class of discrete objects in arbitrary dimensions. The standard model is called a discrete analytical model because the discrete objects (points, $m$-flats, simplices) are defined analytically by inequalities. The analytical definition is independent of the number of discrete points of the object. For instance, a 3D standard triangle is defined by 17 or less inequalities independently of its size.

The model we propose has many interesting properties. The model is geometrically consistent: for instance, the vertices of a 3D standard polygon are 3D standard points, the edges of a 3D standard polygon are 3D standard line segments and the 3D standard polygon is a piece of a 3D standard plane. It has 
been shown that the standard model is in fact a 0-discretisation of Brimkov, Andres and Barneva [12] and therefore is $(n-1)$-connected and tunnel-free. In $3 \mathrm{D},(n-1)$-connectivity in our notations corresponds to the classical 6 connectivity. Contrary to the supercover model, from which it derives, the standard objects are bubble-free. One of the problems of the supercover model is that it is not topologically consistent. A supercover $m$-flat is always $(n-1)$ connected but sometimes it has simple points (located on so-called bubbles on the object). This makes the model difficult to use in practice $[14,15]$. For instance, a supercover of a Euclidean $\mathrm{nD}$ point can be composed of any $2^{i}$ discrete points, $0 \leq i \leq n$. A standard $m$-flat is almost identical to the supercover $m$-flat, it remains $(n-1)$-connected and tunnel-free, except for the simple points in the bubbles that are removed. The standard digitization of a nD Euclidean point is always composed only of one discrete point. Finally, the standard model has a very important property in the framework of discrete modelling: $\mathbb{S} t(F \cup G)=\mathbb{S} t(F) \cup \mathbb{S} t(G)$. This means that, for instance, the definition of the standard 3D polygon is sufficient to define the standard model of an arbitrary Euclidean polygonal 3D object.

The definition of the standard model is derived from the supercover model $[2,5,6,14,15,31,7]$. A standard object is obtained by a simple rewriting process of the inequalities defining analytically a supercover object [7]. A supercover linear object is defined by a set of inequalities " $\sum_{i=1}^{n} a_{i} X_{i} \leq a_{0}$ ". The simple points in the bubbles are points that verify " $\sum_{i=1}^{n} a_{i} X_{i}=a_{0} "$. In order to remove the simple points, and thus bubbles, some of the inequalities need simply to be rewritten into " $\sum_{i=1}^{n} a_{i} X_{i}<a_{0} "$. The selection of inequalities that are modified is based on an orientation convention. Depending on the orientation of the half-space, the corresponding inequality is modified or not. 
In section 2, we introduce our notations and the principal properties of the supercover model on which the standard model is based. In section 3 the standard model is introduced and defined. We start, in section 3.1, by explaining why such a "heavy" mathematical machinery is necessary to define $(n-1)$ connected discrete objects. We show in particular why a classical, misleading, approach does not work. In section 3.2, we explain the basic ideas behind the standard model. In section 3.3, we introduce the orientation convention that forms the basis of the definition of the standard model. The standard model is defined for all linear primitives in dimension $\mathrm{n}$ in section 3.4. The properties of the standard primitives, especially the tunnel-freeness and the $(n-1)$-connectivity, are presented in section 3.5. In section 4 , we examine the different classes of standard linear objects to see how the definition is translated in practice and how the different inequalities defining the objects are established. Conclusion and several perpectives are presented in section 5 .

\section{Preliminaries}

\subsection{Basic notations in discrete geometry}

Most of the following notations correspond to those given by Cohen and Kaufman in [14,15] and those given by Andres in [7]. We provide only a short recall of these notions.

Let $\mathbb{Z}^{n}$ be the subset of the $n \mathrm{D}$ Euclidean space $\mathbb{R}^{n}$ that consists of all the integer coordinate points. A discrete (resp. Euclidean) point is an element of $\mathbb{Z}^{n}$ (resp. $\left.\mathbb{R}^{n}\right)$. A discrete (resp. Euclidean) object is a set of discrete (resp.

Euclidean) points. A discrete inequality is an inequality with coefficients in $\mathbb{R}$ 


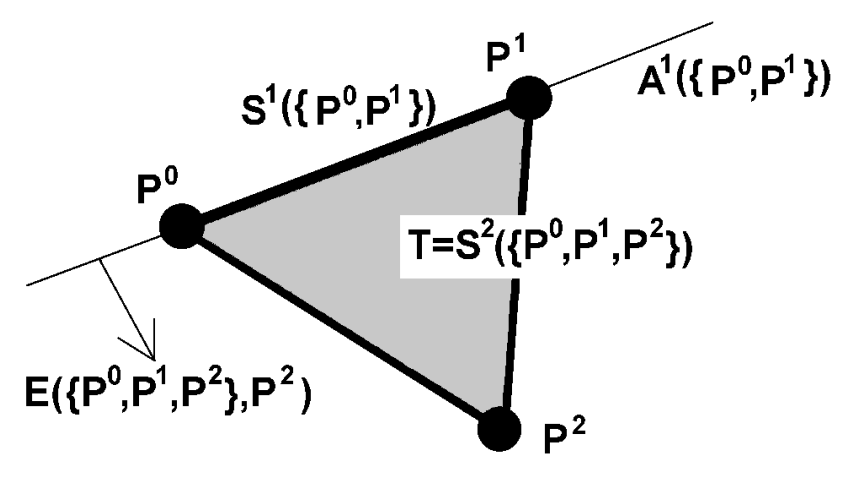

Fig. 1. Triangle $T=S^{2}\left(\left\{P^{0}, P^{1}, P^{2}\right\}\right)$, edge $S^{1}\left(\left\{P^{0}, P^{1}\right\}\right)$, straight line $A^{1}\left(\left\{P^{0}, P^{1}\right\}\right)$ and half-space $E\left(\left\{P^{0}, P^{1}, P^{2}\right\}, P^{2}\right)$

from which we retain only the integer coordinate solutions. A discrete analytical object is a discrete object defined by a finite set of discrete inequalities. An $m$-flat is a Euclidean affine subspace of dimension $m$.

Let us consider a set $P$ of $m+1$ linearly independent Euclidean points $P^{0}, \ldots, P^{m}$. We denote $A^{m}(P)$ the $m$-flat induced by $P$ (i.e. the $m$-flat containing $P$ ). We denote $S^{m}(P)$ the geometrical simplex of dimension $m$ in $\mathbb{R}^{n}$ induced by $P$ (i.e. the convex hull of $P$ ). For $S=S^{m}(P)$ a geometrical simplex, we denote $\bar{S}=A^{m}(P)$ the corresponding $m$-flat. For a $n$-simplex $S=S^{n}(P)$, we denote $E\left(S, P^{i}\right)$ the half-space of boundary $A^{n-1}\left(P \backslash P^{i}\right)$ that contains $P^{i}$ (see figure 1).

We denote $p_{i}$ the $i$-th coordinate of a point or vector $p$. Two discrete points $p$ and $q$ are $k$-neighbours, with $0 \leq k \leq n$, if $\left|p_{i}-q_{i}\right| \leq 1$ for $1 \leq i \leq n$, and $k \leq n-\sum_{i=1}^{n}\left|p_{i}-q_{i}\right|$. The voxel $\mathbb{V}(p) \subset \mathbb{R}^{n}$ of a discrete $n \mathrm{D}$ point $p$ is defined by $\mathbb{V}(p)=\left[p_{1}-\frac{1}{2}, p_{1}+\frac{1}{2}\right] \times \cdots \times\left[p_{n}-\frac{1}{2}, p_{n}+\frac{1}{2}\right]$. For a discrete object $F$, $\mathbb{V}(F)=\bigcup_{p \in F} \mathbb{V}(p)$. We denote $\sigma^{n}$ the set of all the permutations of $\{1, \ldots, n\}$. Let us denote $\mathbb{J}_{m}^{n}$ the set of all the strictly growing sequences of $m$ integers all between 1 and $n: \mathbb{J}_{m}^{n}=\left\{j \in \mathbb{Z}^{m} \mid 1 \leq j_{1}<j_{2}<\ldots<j_{m} \leq n\right\}$. This defines a 
set of multi-indices.

Let us consider an object $F$ in the $n$-dimensional Euclidean space $\mathbb{R}^{n}$, with $n>1$

The orthogonal projection is defined by:

$$
\begin{aligned}
& \pi_{i}(F)=\left\{\left(q_{1}, \ldots, q_{i-1}, q_{i+1}, \ldots, q_{n}\right) \mid q \in \mathbb{R}^{n}\right\}, \text { for } 1 \leq i \leq n \\
& \pi_{j}(F)=\left(\pi_{j_{1}} \circ \pi_{j_{2}} \circ \cdots \circ \pi_{j_{m}}\right)(F), \text { for } j \in \mathbb{J}_{m}^{n} .
\end{aligned}
$$

The orthogonal extrusion is defined by:

$$
\varepsilon_{j}(F)=\pi_{j}^{-1}\left(\pi_{j}(F)\right), \text { for } j \in \mathbb{J}_{m}^{n} \text {. }
$$

Example: Let us consider the set of points $P=\left\{P^{0}(0,0,0), P^{1}(9,1,1), P^{2}(3,8,4)\right\}$.

The corresponding simplex $T=S^{2}(P)$ is a 3D triangle. The orthogonal projection $\pi_{2}(T)=S^{2}\left(\pi_{2}(P)\right)=S^{2}(\{(0,0),(9,1),(3,4)\})$ is a $2 \mathrm{D}$ triangle. The orthogonal extrusion $\varepsilon_{2}(T)=\{(0, t, 0),(9, t, 1),(3, t, 4) \mid t \in \mathbb{R}\}$ is a $3 \mathrm{D}$ Euclidean object defined by 3 half-spaces.

We define an axis arrangement application $r_{j}$, for $j \in \mathbb{J}_{m}^{n}$, by:

$$
\begin{aligned}
r_{j} & : \mathbb{R}^{n} \rightarrow \mathbb{R}^{n} \\
x & \mapsto\left(x_{\sigma_{j}(1)}, x_{\sigma_{j}(2)}, \ldots, x_{\sigma_{j}(n)}\right)
\end{aligned}
$$

where the permutation $\sigma_{j} \in \sigma^{n}$ is defined by:

$$
\sigma_{j}=\left\{\begin{array}{c}
\text { for } 1 \leq i \leq m, \sigma_{j}\left(j_{i}\right)=i \\
\text { else, for } m<i \leq n, \sigma_{j}\left(k_{r}\right)=i
\end{array}\right.
$$


so that $k_{r}<k_{r+1}$ and $k_{r} \neq j_{s}$ for all $1 \leq r \leq n-m$ and for all $1 \leq$ $s \leq m$. The axis arrangement application has been specifically designed so that it verifies the two following properties: $\pi_{j}(F)=\pi_{(1,2, \ldots, m)}\left(r_{j}^{-1}(F)\right)$ and $\varepsilon_{j}(F)=r_{j}\left(\varepsilon_{(1,2, \ldots, m)}\left(r_{j}^{-1}(F)\right)\right)$ for all $F$ in $\mathbb{R}^{n}$ and $j \in \mathbb{J}_{m}^{n}$.

Example: Let us consider the $5 \mathrm{D}$ point $P(1,2,3,4,5)$ and $j=(2,4) \in \mathbb{J}_{2}^{5}$. The corresponding axis arrangement application is defined by $r_{(2,4)}: x \mapsto$ $\left(x_{3}, x_{1}, x_{4}, x_{2}, x_{5}\right)$ and $r_{(2,4)}^{-1}: x \mapsto\left(x_{2}, x_{4}, x_{1}, x_{3}, x_{5}\right)$. The orthogonal projection verifies $\pi_{(2,4)}(P)=\pi_{(1,2)}\left(r_{(2,4)}^{-1}(P)\right)=\pi_{(1,2)}(2,4,1,3,5)=(1,3,5)$.

The orthogonal extrusion verifies $\varepsilon_{(2,4)}(1,3,5)=r_{(2,4)}\left(\varepsilon_{(1,2)}\left(r_{(2,4)}^{-1}(P)\right)\right)$ $=r_{(2,4)}\left(\varepsilon_{(1,2)}(2,4,1,3,5)\right)=r_{(2,4)}\left(\pi_{(1,2)}^{-1}(1,3,5)\right)$ and therefore $\varepsilon_{(2,4)}(1,3,5)=r_{(2,4)}\left(\left\{(t, u, 1,3,5) \mid(t, u) \in \mathbb{R}^{2}\right\}\right)=\left\{(1, t, 3, u, 5) \mid(t, u) \in \mathbb{R}^{2}\right\}$.

\subsection{Geometric properties of the Supercover}

A discrete object $G$ is a cover of a Euclidean object $F$ if $F \subset \mathbb{V}(G)$ and $\forall p \in G, \mathbb{V}(p) \cap F \neq \varnothing$. The supercover $\mathbb{S}(F)$ of a Euclidean object $F$ is defined by $\mathbb{S}(F)=\left\{p \in \mathbb{Z}^{n} \mid \mathbb{V}(p) \cap F \neq \varnothing\right\}$ (see Figure 2a). $\mathbb{S}(F)$ is by definition a cover of $F$. It is easy to see that if $G$ is a cover of $F$ then $G \subset \mathbb{S}(F)$. The supercover of $F$ can be defined in different ways: $\mathbb{S}(F)=\left(F \oplus B^{\infty}\left(\frac{1}{2}\right)\right) \cap \mathbb{Z}^{n}=$ $\left\{p \in Z^{n} \mid d^{\infty}(p, F) \leq \frac{1}{2}\right\}$ (see Figure 2b) where $B^{\infty}(r)$ if the ball centered on the origin, of radius $r$ for the distance $d^{\infty}$. This links the supercover to mathematical morphology $[29,31,7,26]$.

The supercover has many properties. Let us consider two Euclidean objects $F$ and $G$, and a multi-index $j \in \mathbb{J}_{m}^{n}$, then: $\mathbb{S}(F)=\bigcup_{\alpha \in F} \mathbb{S}(\alpha), \mathbb{S}(F \cup G)=$ $\mathbb{S}(F) \cup \mathbb{S}(G)$, if $F \subset G$, then $\mathbb{S}(F) \subset \mathbf{S}(G)$. These properties are well 


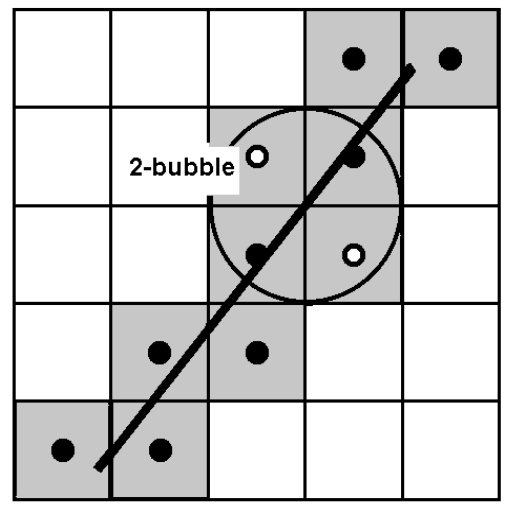

(a)

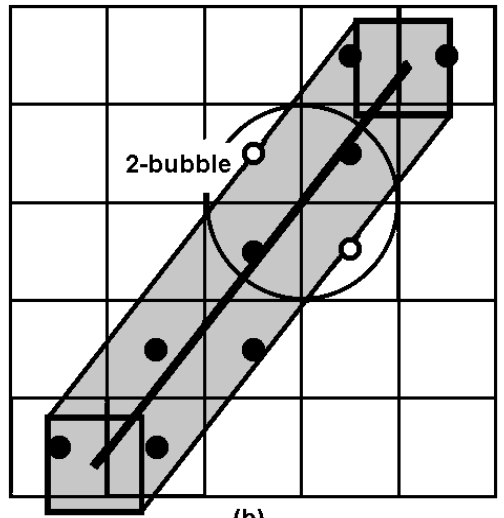

(b)

Fig. 2. Supercover definitions.

known $[14,15]$. The following properties are more recent and are useful in the framework of this paper: $\mathbb{S}(F \times G)=\mathbb{S}(F) \times \mathbb{S}(G), r_{j}(\mathbb{S}(F))=\mathbb{S}\left(r_{j}(F)\right)$, $\pi_{j}(\mathbb{S}(F))=\mathbb{S}\left(\pi_{j}(F)\right)$ and $\varepsilon_{j}(\mathbb{S}(F))=\mathbb{S}\left(\varepsilon_{j}(F)\right)=r_{j}\left(\mathbb{Z}^{m} \times \mathbb{S}\left(\pi_{j}(F)\right)\right)[7]$.

\section{Definition 1 (Bubble)}

A $k$-bubble, with $1 \leq k \leq n$, is the supercover of a Euclidean point that has exactly $k$ half-integer coordinates.

A half-integer is a real $l+\frac{1}{2}$, with $l$ an integer. A $k$-bubble is formed of $2^{k}$ discrete points. A 2-bubble can be seen in figure $2 \mathrm{a}$ (marked by the black circle). The two white dots are what we call here "simple" points. This corresponds to an extension of the notion of simple points that fits a supercover simplex. A point $P$ belonging to the supercover simplex $S$ is said to be a simple point if it is a simple point for $\bar{S}$ with the classical definition given in section 2.1.

\section{Definition 2 (Bubble-free)}

The cover of an $m$-flat is said to be bubble-free if it has no $k$-bubbles for $k>m$.

The cover of a simplex $S$ is said to be bubble-free if $\bar{S}$ is bubble-free. 
There are two types of bubbles in the supercover of an $m$-flat $F$. The $k$-bubbles, for $k \leq m$, are discrete points that are part of all the covers of $F$. If we remove any of these points, the discrete object is not a cover anymore. In the $k$ bubbles, for $k>m$, there are discrete points that are "simple" points. The aim of this paper is to propose discrete analytical objects that are bubble-free and $(n-1)$-connected by removing some of the simple points. In figure $2 \mathrm{a}$, by removing one of the two simple points, we obtain a bubble-free, 1-connected discrete 2D line segment.

Lemma 1 A discrete point $p$ belongs to a $k$-bubble, $k>m$, of the supercover of an $m$-flat $F$ if and only if there exists a point $\alpha \in F$ with $k$ half-integer coordinates such that $p \in \mathbb{S}(\alpha)$.

The proof of this lemma is obvious.

\section{Standard Model}

The aim of this paper is to propose a new cover class, called the standard cover. The standard cover is so far only defined for linear objects in all dimensions. The discrete analytical model has been designed to conserve most of the properties of the supercover, to be bubble-free and $(n-1)$-connected. The supercover model has almost all the properties we are looking for: tunnel-

freeness, $(n-1)$-connectivity, stability for union, etc. The only property that is missing is the bubble-freeness. Some supercover objects have simple points. The model is therefore not topologically consistent and this is a problem for several applications such as, for instance, polygonalization. For this reason several attempts have been made to modify the supercover discretization by 
modifying the definition of a pixel $[27,14,15]$. We show in the following section that such attempts can't work. In our approach, presented in section 3.2, we explain how, by studying the analytical description of linear objects, it is possible to remove selectively the simple points in the supercover model while preserving the modeling properties. In the section that follow the standard model and its properties are introduced.

\subsection{What does not work with the classical approach}

Several unsuccessful attempts have been made to define discrete objects that have supercover type modeling properties with bubble-freeness and $(n-1)$ connectivity properties $[27,14,15]$. All these ideas basically modify, in various ways, the definition of a voxel in order to avoid bubbles. We give here a simple such example and show why it does not work that way (see [14] for some other examples). In Figure 3, the pixel definition has been changed. A pixel is now formed of the SW vertex (black disk), the two corresponding edges (bold edges) and its' interior. The three other vertices and two other edges do not belong to the pixel. This definition derives that $\bigcup_{p \in \mathbb{Z}^{n}} \mathbb{V}(p)=\mathbb{R}^{n}$ with $\mathbb{V}(p) \cap \mathbb{V}(q)=\varnothing$ for $p \neq q$. The discretisation of a discrete line is necessarily bubble-free. However, as we see in figure 3, the discretised line $x_{1}-x_{2}=0$ is not 1-connected. In fact, it has been shown as early as in 1970 [27], that no change in the definition of the pixel or voxel can lead to a correct solution. This means that a simple pixel definition modification avoids bubbles but creates primitives that aren't topologically consistent. This makes such a model useless for applications such as polygonalization. Tunnel-freeness property is also lost with such an approach. 

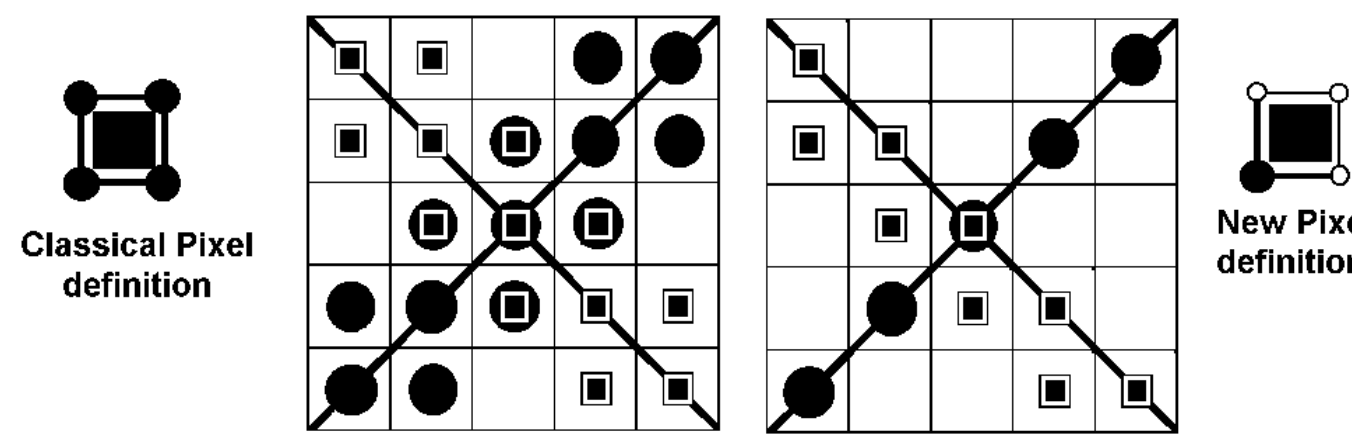

New Pixel definition

Fig. 3. Supercover discretisation of $x_{1}-x_{2}=0$ and $x_{1}+x_{2}=0$ with classical and modified pixel definition.

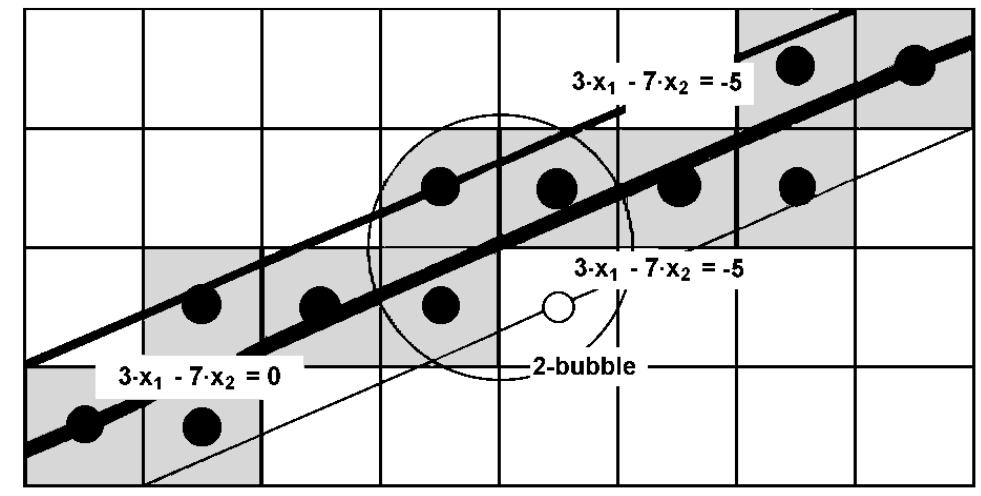

Fig. 4. Standard and Supercover straight line. The black points belong to both line. The white point belongs only to the supercover.

\subsection{Standard model approach : a modification of the supercover definition}

The discrete analytical description of the supercover of a linear convex is defined as intersection of half-spaces defined by discrete inequalities $\sum_{i=1}^{n} a_{i} x_{i} \leq$ $a_{0}[2,5-7]$. A linear concave object is simply considered as union of convexes. The orientation of each half-space is checked with an orientation convention and depending on it, its inequality " $\sum_{i=1}^{n} a_{i} x_{i} \leq a_{0}$ " remains unchanged or is replaced by " $\sum_{i=1}^{n} a_{i} x_{i}<a_{0}$ ". 
Let us give a simple example, the $2 \mathrm{D}$ straight line $D: 3 x_{1}-7 x_{2}=0$ shown in

figure 4, to illustrate why and how this works. The general case in dimension $n$ works exactly in the same way. The supercover of the Euclidean line $D$ is described by the two inequalities $\mathbb{S}(D)=\left\{\left(x_{1}, x_{2}\right) \in \mathbb{Z}^{2} \mid-5 \leq 3 x_{1}-7 x_{2} \leq 5\right\}$. A bubble occurs only when the straight line $D$ contains half-integer coordinate points. We have then (and only then) discrete points verifying on one side $3 x_{1}-7 x_{2}=-5$ and on the other side $3 x_{1}-7 x_{2}=5$. All these points are simple points. Removing the points on one side only leads to a discrete straight line that is 1-connected, separating, 1-minimal and bubble-free. This can be done simply by replacing a " $\leq$ " by a " $<$ " for one of the two inequalities in the supercover analytical description. In the case of Figure 4, we have $\mathbb{S} t(D)=\left\{\left(x_{1}, x_{2}\right) \in \mathbb{Z}^{2} \mid-5 \leq 3 x_{1}-7 x_{2}<5\right\}$. The change is based on an orientation convention. Opposing half-spaces such as " $3 x_{1}-7 x_{2} \leq 5$ " and " $-3 x_{1}+7 x_{2} \leq 5 "$ have a different orientation in this convention and thus only one of them will have its" " $\leq$ " changed into " $<$ ". This ensures that only one simple point for the $2 \mathrm{D}$ line will be removed.

\subsection{Orientation convention}

The standard model, contrary to the supercover, is not unique $[7,9]$. For instance, in example of Figure 4, one of two possible simple points can be removed. Each selection leads to another standard model definition. It depends on the orientation convention selection. One orientation convention per dimension $\mathbb{R}^{m}, m>0$, is required. This selection must then remain unchanged for all the primitives handled. The selection of an orientation convention per dimension has to be coherent with the operator $\pi$. The property $\mathbb{S} t\left(\pi_{j}(F)\right)=$ 
$\pi_{j}(\mathbb{S} t(F))$ for the operator $\pi$ should be verified. If this is not the case, the modelling properties won't be verified (such as $\mathbb{S} t(F \cup G)=\mathbb{S} t(F) \cup \mathbb{S} t(G)$, etc.). In general, with arbitrary orientation conventions there is no reason for this property to be verified. We propose a set of orientation conventions, denoted $O^{n}$ and called the basic orientation conventions. The basic orientation conventions verify the above mentioned property.

\section{Definition 3 (Standard orientation)}

Let us consider a discrete analytical half-space $E: \sum_{i=1}^{n} C_{i} X_{i} \leq B$ and the basic orientation convention $O^{n}$. We say that $E$ has a standard orientation if :

- $C_{1}>0$

- or if $C_{1}=0$ and $C_{2}>0$;

- :

- or if $C_{1}=\cdots=C_{n-1}=0$ and $C_{n}>0$.

If $E$ has not a standard orientation then we say that $E$ has a supercover orientation.

We consider from now on, without loss of generality, only the basic orientation conventions for all $n>0$. All the standard primitives are defined with these basic orientation conventions. The basic orientation conventions are coherent with respect to the operators $\pi$. After $\pi_{j}$, for $j \in \mathbb{J}_{m}^{n}$, the orientation convention $O^{n}$ in $\mathbb{R}^{n}$ becomes $O^{n-m}$ in $\mathbb{R}^{n-m}$. 


\subsection{Standard model definition}

All the elements required to define the standard discretisation model of linear objects in $\mathbb{R}^{n}$ are available:

\section{Definition 4 (Standard Model)}

Let $F$ be a linear Euclidean object in $\mathbb{R}^{n}$ whose supercover is described analytically by a finite set of inequalities $F_{k}: \sum_{i=1}^{n} C_{i, k} X_{i} \leq B_{k}$. The standard model $\mathbb{S} t(F)$ of $F$, for the basic orientation convention $O^{n}$, is the discrete object described analytically by a finite set of discrete inequalities $F_{k}^{\prime}$ obtained by substituting each inequality $F_{k}$ by $F_{k}^{\prime}$ defined as follows:

- If $F_{k}$ has a standard orientation then $F_{k}^{\prime}: \sum_{i=1}^{n} C_{i, k} X_{i}<B_{k}$;

- else $F_{k}^{\prime}: \sum_{i=1}^{n} C_{i, k} X_{i} \leq B_{k}$.

This definition is algorithmically easy to set up. Once a discrete analytical description of an object is available, the transition from the supercover model to the standard model and vice-versa is trivial.

\subsection{Geometric properties of the Standard model}

In this section, some properties of the standard model are presented. These properties are very important for the derivation of our model description. Let us consider a Euclidean linear object $F$ of topological dimension $m$ in $\mathbb{R}^{n}$. We have by definition $\mathbb{S} t(F) \subset \mathbb{S}(F)$ even more precisely, if $p \in \mathbb{S}(F) \backslash \mathbb{S} t(F)$, then $d^{\infty}(p, F)=\frac{1}{2}$. A standard object is a supercover object from which some

discrete points have been removed. These points are all at a distance $\frac{1}{2}$ from 
the Euclidean primitive. We have $\mathbb{S} t(F)=\mathbb{S}(F)$ if no point, with at least $m+1$ half-integer coordinates, belongs to the boundary of $F$. The differences between the supercover of $F$ and the standard model of $F$ are located in the $k$-bubbles of $F$, for $k>m$. Figure 4 illustrates this in dimension 2. One of the immediate consequences of this last property, is that the standard model remains a cover: $F \subset \mathbb{V}(\mathbb{S} t(F))$. That is why the standard model is also sometimes called standard cover [33,31].

The standard model retains most of the set properties of the supercover. It is easy to deduce from definition 4, that if we consider two Euclidean linear objects $F$ and $G$ in $\mathbb{R}^{n}$, then:

$$
\begin{gathered}
\mathbb{S} t(F \cup G)=\mathbb{S} t(F) \cup \mathbb{S} t(G) ; \quad \mathbb{S} t(F \cap G) \subset \mathbb{S} t(F) \cap \mathbb{S} t(G) \\
F \subset G \Rightarrow \mathbb{S} t(F) \subset \mathbb{S} t(G) ; \quad \mathbb{S} t(F \times G)=\mathbb{S} t(F) \times \mathbb{S} t(G) ; \\
\mathbb{S} t\left(\pi_{j}(F)\right)=\pi_{j}(\mathbb{S} t(F)) ; \quad \mathbb{S} t\left(\varepsilon_{j}(F)\right)=\varepsilon_{j}(\mathbb{S} t(F)) .
\end{gathered}
$$

The first property ensures that we'll be able to construct complex discrete objects out of basic elements such as simplices. These last properties are characteristic of correct orientation conventions. The properties are only verified if the orientation conventions are defined for all dimensions lower or equal to $n$ and if they are coherent with respect to the operator $\pi$. This is the case for the basic orientation conventions $O^{k}$, for $k \leq n$.

It is important to notice that, in general, $\mathbb{S} t(F) \neq \bigcup_{\alpha \in F} \mathbb{S} t(\alpha)$. This property of the supercover is not conserved. We have $\mathbb{S} t(F \cup G)=\mathbb{S} t(F) \cup \mathbb{S} t(G)$ for a union of a finite number of objects. This comes simply from the fact that the standard model is not defined for an analytical description that has an infinite 
number of discrete inequalities. One simple example for that is given by the $2 \mathrm{D}$ line $D: x_{1}-x_{2}=0$. The standard model of the line is $\mathbb{S} t(D):-1 \leq x_{1}-x_{2}<1$ while $\bigcup_{\alpha \in F} \mathbb{S} t(\alpha):-1<x_{1}-x_{2}<1$..

One of the main properties of the standard model concerns the connectivity and the tunnel-freeness:

Theorem 2 (connectivity and tunnel-freeness)

Let $F$ be a Euclidean linear object of topological dimension $m$ in $\mathbb{R}^{n}$. Its standard model $\mathbb{S} t(F)$ is $(n-1)$-connected and tunnel-free.

The standard model is a particular case of $k$-discretisations as introduced by Brimkov, Andres and Barneva in [12]. It is shown that the standard model is in fact a 0 -discretisation (Theorem 3 in [12]) and that 0-discretisations are $(n-1)$-connected and tunnel-free (proposition 3 in [11] and theorem 4 in [12]). Another property proved in $[31,33,12]$ is that the standard model minimizes the Hausdorff distance with the Euclidean object.

\section{Description of standard primitives}

We'll examine now the discrete analytical description of the different classes of standard linear primitives (half-space, point, $m$-flat and $m$-simplex) and how they can be computed. Our purpose here is to propose a discretisation scheme that can be used is practical applications. As stated in definition 4, every analytical description of a standard linear primitive is based on the analytical description of a standard half-space. That is the one we present

first. We deduce from it the discrete analytical formulas describing a standard 
point, $m$-flat and $m$-simplex in the sections that follow.

\subsection{Standard Half-space}

The standard half-space is given by :

\section{Proposition 3 (Standard half-space)}

Let us consider a Euclidean half-space $E: \sum_{i=1}^{n} C_{i} X_{i} \leq B$. The standard model $\mathbb{S} t(E)$ of $E$, according to an orientation convention, is analytically described by:

- If $E$ has a standard orientation then $\mathbb{S} t(E)=\left\{p \in \mathbb{Z}^{n} \mid \sum_{i=1}^{n} C_{i} p_{i}<B+\frac{\sum_{i=1}^{n}\left|C_{i}\right|}{2}\right\}$;

- else $\mathbb{S} t(E)=\left\{p \in \mathbb{Z}^{n} \mid \sum_{i=1}^{n} C_{i} p_{i} \leq B+\frac{\sum_{i=1}^{n}\left|C_{i}\right|}{2}\right\}$.

The proposition is an immediate extension to dimension $n$ of results on the supercover $[2,4,5,7]$ and of definition 4 .

\subsection{Standard point}

The analytical description of a standard point can easily be deduced from the one of the standard half-space. It is however interesting to notice that the standard discretisation of a Euclidean point is always composed of one and only one discrete point contrary to what happens with a supercover discretization of a Euclidean point that can be formed of $2^{k}$ points, $0 \leq k \leq n$ (in case of a $k$-bubble).

\section{Proposition 4 (Standard point)}




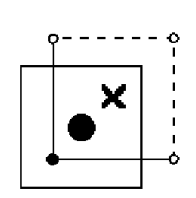

(a)

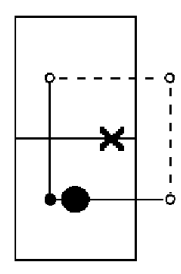

(b)

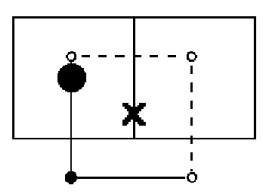

(c)

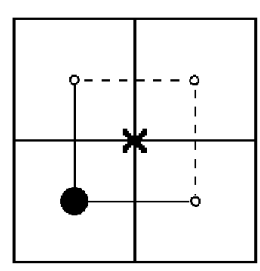

(d)

Fig. 5. Different configurations of 2D standard points. A standard point is formed of only one discrete point.

Let us consider a Euclidean point $\alpha \in \mathbb{R}^{n}$ and the basic orientation convention $O^{n}$. The standard model $\mathbb{S} t(\alpha)$ of $\alpha$ is the discrete point:

$$
\mathbb{S} t(\alpha)=\left(\left\lceil\alpha_{1}-\frac{1}{2}\right\rceil, \ldots,\left\lceil\alpha_{n}-\frac{1}{2}\right\rceil\right)
$$

The proof is obvious. In figure 5, the cross represents the Euclidean point. The black dot represents the corresponding discrete standard point. The square with the doted lines represent the zone covered by the 4 inequalities corresponding to the analytical description of a standard point.

\subsection{Standard $m$-flat}

One of the consequences of the properties $\mathbb{S} t\left(\pi_{j}(F)\right)=\pi_{j}(\mathbb{S} t(F))$ and $\mathbb{S} t\left(\varepsilon_{j}(F)\right)=$ $\varepsilon_{j}(\mathbb{S} t(F))$ is that the formulas that lead to the discrete analytical description of a standard $m$-flat or of a standard $m$-simplex are simple transpositions of the formulas that have been established for the supercover [7].

\section{Proposition 5 (Standard $m$-flat)}

Let us consider an $m$-flat $F$ in $\mathbb{R}^{n}$ and the basic orientation conventions $O^{k}$, for all $k>0$. 
a) If $F$ is a 0 -flat in $\mathbb{R}^{n}$, we apply proposition 4,

b) If $F$ is a $(n-1)$-flat, we apply twice proposition 3,

c) else the analytical description of the standard model of $F$ is given by:

$$
\mathbb{S} t(F)=\bigcap_{j \in \mathbb{J}_{n-1-m}^{n}} \mathbb{S} t\left(\varepsilon_{j}(F)\right)=\bigcap_{j \in \mathbb{J}_{n-1-m}^{n}} r_{j}\left(\mathbb{Z}^{m} \times \mathbb{S} t\left(\pi_{j}(F)\right)\right)
$$

We reapply then, recursively, the corresponding cases a), b) or c) on $\pi_{j}(F)$ for all $j \in \mathbb{J}_{n-1-m}^{n}$.

This proposition is composed of several steps that lead to the analytical description of the standard $m$-flat. Let us discuss step c). The formula $\mathbb{S} t(F)=$ $\bigcap_{j \in \mathbb{J}_{n-1-m}^{n}} \mathbb{S} t\left(\varepsilon_{j}(F)\right)$ alone is not sufficient to describe the standard $m$-flat, with $0<m<n-1$, since $\varepsilon_{j}(F)$ is not necessarily a hyperplane in $\mathbb{R}^{n}$. We might even have $F=\varepsilon_{j}(F)$ for some $j \in \mathbb{J}_{n-1-m}^{n}$. The way around this problem is to examine $\pi_{j}(F)$ in $\mathbb{R}^{m+1}$. The new orientation convention for $\mathbb{R}^{m+1}$ after $\pi_{j}$ is $O^{m+1}$.

We have different cases that occur:

- If $\pi_{j}(F)$ is a hyperplane in $\mathbb{R}^{m+1}$ then $\varepsilon_{j}(F)$ is a hyperplane in $\mathbb{R}^{n}$. We do not actually need to consider $\pi_{j}(F)$. We can directly use case b). It is easy to see that we obtain the same result by applying directly proposition 3 on $\varepsilon_{j}(F)$ or by applying $r_{j}\left(\mathbb{Z}^{m} \times \mathbb{S} t\left(\pi_{j}(F)\right)\right)$.

- If $\pi_{j}(F)$ is a point in $\mathbb{R}^{m+1}$ then we consider case a) in $\mathbb{R}^{m+1}$, with the basic orientation convention, and formula $r_{j}\left(\mathbb{Z}^{m} \times \mathbb{S} t\left(\pi_{j}(F)\right)\right)$.

- If $\pi_{j}(F)$ is a $k$-flat, $0<k<m$, in $\mathbb{R}^{m+1}$ then we consider again case c), with the basic orientation convention. We have, by definition, $\mathbb{S} t\left(\pi_{j}(F)\right)=$ $\bigcap_{j^{\prime} \in \mathbb{J}_{m-1}^{m+1}} \mathbb{S} t\left(\varepsilon_{j^{\prime}}\left(\pi_{j}(F)\right)\right)$. We repeat the operation described in case c) for $\pi_{j^{\prime}}\left(\pi_{j}(F)\right)$ in $\mathbb{R}^{k+1}$. 
We know that this process ends since each time we repeat case c) we consider a new object in a space of strictly lower dimension. The following example illustrates how this definition works in practice:

Example : Let us consider the 3D line $F=\{t(6,7,3) \mid t \in \mathbb{R}\}$. The supercover of $F$ contains a 2-bubble. We are in case c) of proposition 5 and thus

$$
\mathbb{S} t(F)=\bigcap_{i \in \mathbb{J}_{1}^{3}} \mathbb{S} t\left(\varepsilon_{i}(F)\right)=\mathbb{S} t\left(\varepsilon_{1}(F)\right) \cap \mathbb{S} t\left(\varepsilon_{2}(F)\right) \cap \mathbb{S} t\left(\varepsilon_{3}(F)\right)
$$

We have $\pi_{1}(F)=\{t(7,3) \mid t \in \mathbb{R}\}$ which is a 1-flat in $\mathbb{R}^{2}$ and therefore case b) of proposition 5 applies. We have $\pi_{1}(F)=\left\{X \in \mathbb{R}^{2} \mid 3 X_{1}-7 X_{2}=0\right\}$ that corresponds to two haf-spaces " $3 X_{1}-7 X_{2} \leq 0 "$ and " $-3 X_{1}+7 X_{2} \leq$ $0 "$. The half-space " $3 X_{1}-7 X_{2} \leq 0 "$ has a standard orientation (definition 3). The standard analytical description is therefore " $3 p_{1}-7 p_{2}<5 "$. The half-space " $-3 X_{1}+7 X_{2} \leq 0 "$ has a supercover orientation and its standard description is thus " $-3 p_{1}+7 p_{2} \leq 5 "$. We have therefore $\mathbb{S} t\left(\pi_{1}(F)\right)=$ $\left\{p \in \mathbb{Z}^{2} \mid-5 \leq 3 p_{1}-7 p_{2}<5\right\}$. The analytical description in dimension 3 is obtained with the formula $\mathbb{S} t\left(\varepsilon_{1}(F)\right)=r_{1}\left(\mathbb{Z} \times \mathbb{S} t\left(\pi_{1}(F)\right)\right)$. The axis renumerotation is $r_{1}: x \mapsto\left(x_{1}, x_{2}, x_{3}\right)$.

We have therefore $\mathbb{S} t\left(\varepsilon_{1}(F)\right)=\left\{p \in \mathbb{Z}^{3} \mid-5 \leq 0 p_{1}+3 p_{2}-7 p_{3}<5\right\}$. In the same way we obtain $\mathbb{S} t\left(\varepsilon_{2}(F)\right)$ and $\mathbb{S} t\left(\varepsilon_{3}(F)\right)$ :

As a final result, we have a discrete analytical description of the standard 

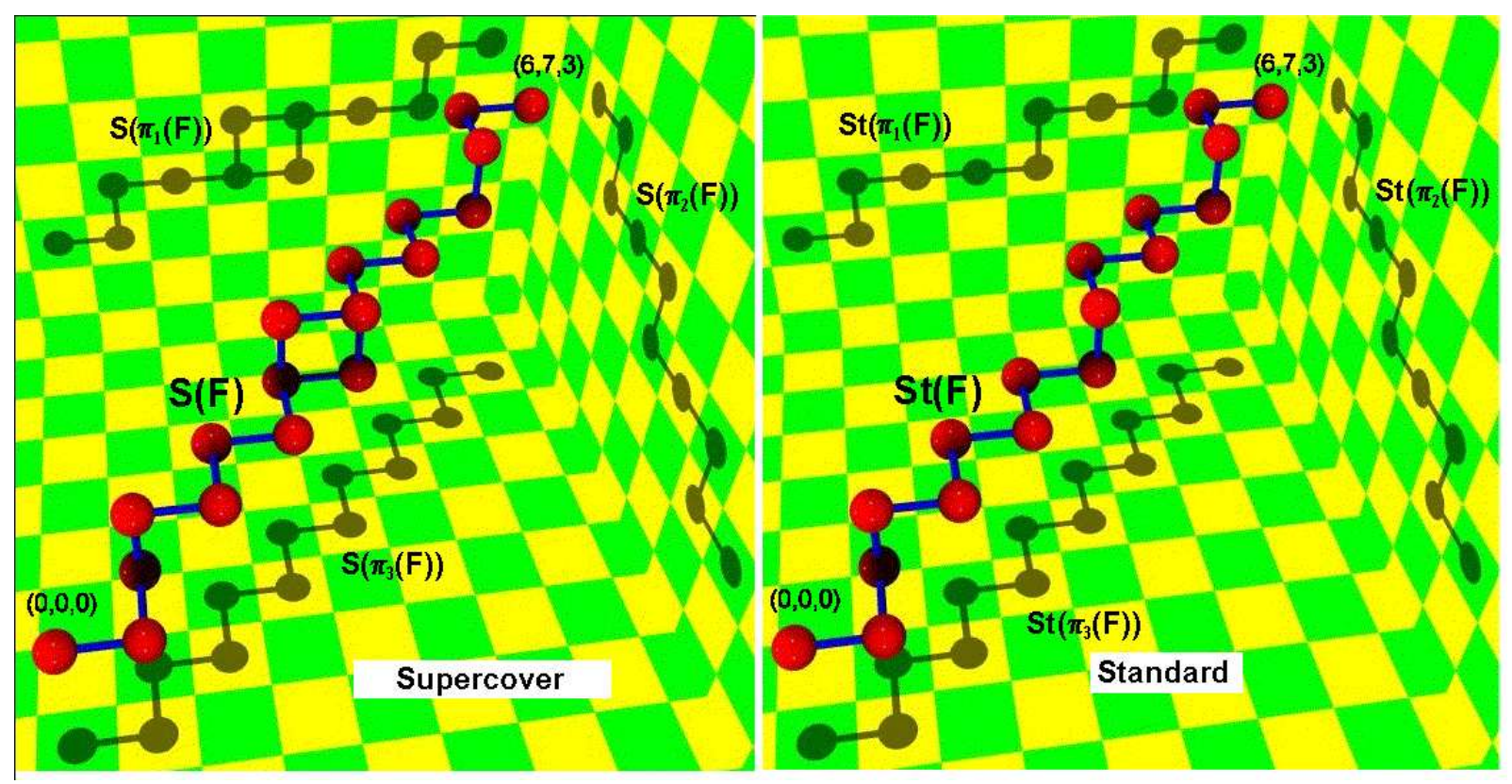

Fig. 6. Supercover and Standard 3D line.

cover of $F$ :

$$
\mathbb{S} t(F)=\left\{p \in \mathbb{Z}^{3} \mid \begin{array}{c}
-5 \leq 3 p_{2}-7 p_{3}<5 \\
-3 / 2 \leq p_{1}-2 p_{3}<3 / 2 \\
-13 / 2 \leq 7 p_{1}-6 p_{2}<13 / 2
\end{array}\right\}
$$

Notice that here $\mathbb{S} t\left(\varepsilon_{2}(F)\right)=\mathbb{S}\left(\varepsilon_{2}(F)\right)$ and $\mathbb{S} t\left(\varepsilon_{3}(F)\right)=\mathbb{S}\left(\varepsilon_{3}(F)\right)$. The only difference between the standard line and the supercover line comes from $\varepsilon_{1}(F)$. This is illustrated by figure 6 that shows the supercover and the standard model of $F$.

Notice that the standard 3D line is in general defined as the intersection of three standard 3D planes. Only straight lines aligned with an axis or orthogonal to an axis require less than three planes (two actually) to be describes analytically in the standard model. This is very different from what happens 
in the Euclidean world where two arbitrary, different, planes containing the line are sufficient to describe analytically a 3D line. It is also very different from the classical methods used to defined discrete 3D lines that use only two projections and orthogonal extrusions to define a 3D line $[1,16,17]$.

Example: Let us examine the discrete analytical description of a standard 2-flat in $\mathbb{R}^{5}$. The standard 2-flat $F$ is defined by:

$$
F=\left\{(0,1,2,3,4)+t(1,2,3,4,5)+u(3,3,6,8,10) \in \mathbb{R}^{5} \mid(t, u) \in \mathbb{R}^{2}\right\}
$$

From case c) of proposition 5 we derive that $\mathbb{S} t(F)=\bigcap_{j \in \mathbb{J}_{2}^{5}} \mathbb{S} t\left(\varepsilon_{j}(F)\right)$, thus:

$$
\begin{aligned}
\mathbb{S} t(F) & =\mathbb{S} t\left(\varepsilon_{(1,2)}(F)\right) \cap \mathbb{S} t\left(\varepsilon_{(1,3)}(F)\right) \cap \mathbb{S} t\left(\varepsilon_{(1,4)}(F)\right) \cap \mathbb{S} t\left(\varepsilon_{(1,5)}(F)\right) \\
& \cap \mathbb{S} t\left(\varepsilon_{(2,3)}(F)\right) \cap \mathbb{S} t\left(\varepsilon_{(2,4)}(F)\right) \cap \mathbb{S} t\left(\varepsilon_{(2,5)}(F)\right) \\
& \cap \mathbb{S} t\left(\varepsilon_{(3,4)}(F)\right) \cap \mathbb{S} t\left(\varepsilon_{(3,5)}(F)\right) \cap \mathbb{S} t\left(\varepsilon_{(4,5)}(F)\right)
\end{aligned}
$$

Let us examine the analytical description of some of the $\mathbb{S} t\left(\varepsilon_{j}(F)\right)$ for $j \in \mathbb{J}_{2}^{5}$ :

- We have $\pi_{(1,2)}(F)=\left\{(2,3,4)+t(3,4,5)+u(6,8,10) \in \mathbb{R}^{3} \mid(t, u) \in \mathbb{R}^{2}\right\}$, and after simplification $\pi_{(1,2)}(F)=\left\{(2,3,4)+t(3,4,5) \in \mathbb{R}^{3} \mid t \in \mathbb{R}\right\}$. We can't apply case b) since $\pi_{(1,2)}(F)$ is straight line and not a hyperplane in dimension 3. As a 1 -flat in $\mathbb{R}^{3}$, we reapply case c) and we obtain $\mathbb{S} t\left(\pi_{(1,2)}(F)\right)=$ $\bigcap_{i \in \mathbb{J}_{1}^{3}} \mathbb{S} t\left(\varepsilon_{i}\left(\pi_{(1,2)}(F)\right)\right)$. We are now exactly in the case of the previous example. We obtain the analytical description of a $3 \mathrm{D}$ line: $\mathbb{S} t\left(\pi_{(1,2)}(F)\right)=$ 
$\left\{p \in \mathbb{Z}^{3} \mid \begin{array}{l}-\frac{11}{2} \leq 5 p_{2}-4 p_{3}<\frac{7}{2} \\ -6 \leq 5 p_{1}-3 p_{3}<2 \\ -\frac{9}{2} \leq 4 p_{1}-3 p_{2}<\frac{5}{2}\end{array}\right\}$.

with $r_{(1,2)}: x \mapsto\left(x_{1}, x_{2}, x_{3}, x_{4}, x_{5}\right)$.

- We have $\pi_{(1,3)}(F)=\left\{(1,3,4)+t(2,4,5)+u(3,8,10) \in \mathbb{R}^{3} \mid(t, u) \in \mathbb{R}^{2}\right\}$ which is a plane in $\mathbb{R}^{3}$. We can therefore apply case b) of proposition 5 . The Euclidean analytical description of the $3 \mathrm{D}$ plane is given by $\pi_{(1,3)}(F)=$ $\left\{X \in \mathbb{R}^{3} \mid 4 X_{1}-6 X_{2}+X_{3}+10=0\right\}$. By applying proposition 3 we obtain $\mathbb{S} t\left(\pi_{(1,3)}(F)\right)=\left\{p \in \mathbb{Z}^{3} \mid-\frac{31}{2} \leq 4 p_{1}-6 p_{2}+p_{3} \leq-\frac{9}{2}\right\}$. We have $\mathbb{S} t\left(\varepsilon_{(1,3)}(F)\right)=$ $r_{(1,3)}\left(\mathbb{Z}^{2} \times \mathbb{S} t\left(\pi_{(1,3)}(F)\right)\right)$ with $r_{(1,3)}: x \mapsto\left(x_{1}, x_{3}, x_{2}, x_{4}, x_{5}\right)$. This leads to the discrete analytical description of $\mathbb{S} t\left(\varepsilon_{(1,3)}(F)\right)=\left\{p \in \mathbb{Z}^{5} \mid-\frac{31}{2} \leq 4 p_{2}-6 p_{4}+p_{5}<-\frac{9}{2}\right\}$.

- The same applies to all the other $\mathbb{S} t\left(\varepsilon_{j}(F)\right)$ which leads to the discrete analytical description of $\mathbb{S} t(F)$ : 


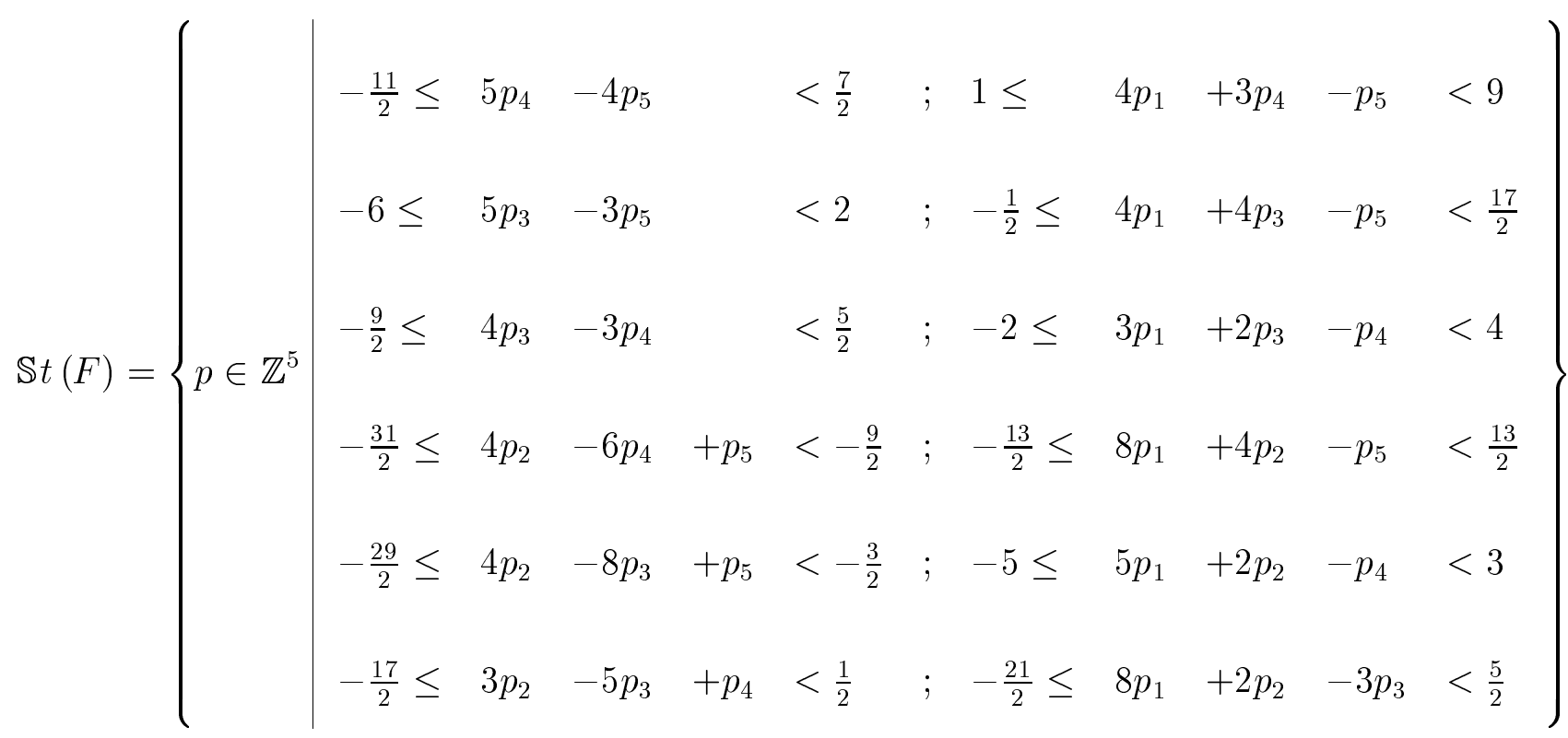

Notice that the discrete analytical description of an $m$-flat in $\mathbb{R}^{n}$ is always described as an intersection of standard hyperplanes $[4,18]$.

\section{4 simplex standard cover}

Let us finish with the formulas describing a standard simplex. These formulas are direct transposition of the formulas obtained for the supercover $[2,5-7,9]$.

Proposition 6 : Let us consider a set $P$ of $m+1$ linearly independent Euclidean point $P^{0}, \ldots, P^{m}$ and the corresponding simplex $S=S^{m}(P)$. We consider the basic orientation conventions $O^{k}$, for all $k>0$. The standard cover of $S$ is defined by:

a) If $m=n$ then $\mathbb{S} t(S)=\left(\bigcap_{i=0}^{n} \mathbb{S} t\left(E\left(S, P^{i}\right)\right)\right) \cap\left(\bigcap_{j=1}^{n} \mathbb{S} t\left(\varepsilon_{j}(S)\right)\right)$;

b) If $m=n-1$ then $\mathbb{S} t(S)=\mathbb{S} t(\bar{S}) \cap\left(\bigcap_{j=1}^{n} \mathbb{S} t\left(\varepsilon_{j}(S)\right)\right)$;

c) If $m \leq n-2$ then $\mathbb{S} t(S)=\bigcap_{j \in \mathbb{J} n n-m-1}^{n} \mathbb{S} t\left(\varepsilon_{j}(S)\right)$. 


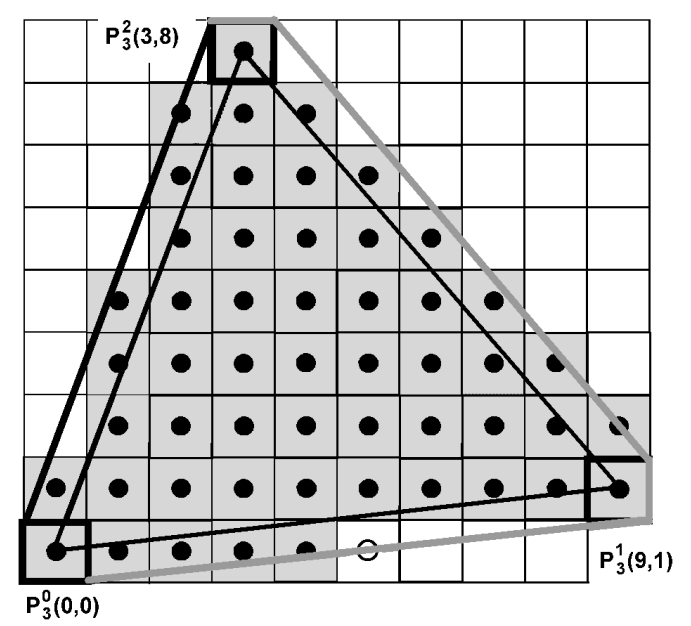

Fig. 7. Standard cover of a $2 \mathrm{D}$ triangle

Let us just recall some notations. If $S$ is a simplex of dimension $m$ then we denote $\bar{S}$ the $m$-flat containing all the points defining $S$. If $S$ is a simplex of dimension $n$ in $\mathbb{R}^{n}$ then we denote $E\left(S, P^{i}\right)$ the half-space that contains $P^{i}$ and of border the $(n-1)$-flat $\overline{\left\{P^{0}, \ldots, P^{i-1}, P^{i+1}, \ldots, P^{m}\right\}}$.

Example: Let us consider now the triangle $T=S^{2}(P)$ with $P=\left\{P^{0}(0,0,0), P^{1}(3,8,4), P^{2}(9,1,1)\right\}$ in $\mathbb{R}^{3}$. We are in case b) of proposition 6: $\mathbb{S} t(T)=\mathbb{S} t\left(A^{2}(P)\right) \cap \mathbb{S} t\left(\varepsilon_{1}(T)\right) \cap \mathbb{S} t\left(\varepsilon_{2}(T)\right) \cap \mathbb{S} t\left(\varepsilon_{3}(T)\right)$.

- The discrete analytical description of the $3 \mathrm{D}$ plane $\mathbb{S} t\left(A^{2}(P)\right)$ is given by $\mathbb{S} t\left(A^{2}(P)\right)=\left\{p \in \mathbb{Z}^{3} \mid-53 \leq 4 p_{1}+33 p_{2}-69 p_{3}<53\right\}$.

- The orthogonal projection $\pi_{3}(T)$ is a $2 \mathrm{D}$ triangle $\pi_{3}(T)=S^{2}\left(P_{3}^{0}, P_{3}^{1}, P_{3}^{2}\right)$ with $P_{3}^{0}=\pi_{3}\left(P^{0}\right)=(0,0), P_{3}^{1}=\pi_{3}\left(P^{1}\right)=(9,1)$ and $P_{3}^{2}=\pi_{3}\left(P^{2}\right)=(3,8)$ (See Figure 7).

- We have a 2-simplex in $\mathbb{R}^{2}$ and case a) of proposition 6 applies: $\mathbb{S} t\left(\pi_{3}(T)\right)=\left(\bigcap_{i=0}^{2} \mathbb{S} t\left(E\left(\pi_{3}(T), P_{3}^{i}\right)\right)\right) \cap\left(\bigcap_{j=1}^{2} \mathbb{S} t\left(\varepsilon_{j}\left(\pi_{3}(T)\right)\right)\right)$. Let us examine each part of the formula.

- The half-space $E\left(\pi_{3}(T), P_{3}^{0}\right)$ is delimited by the straight line $A^{1}\left(\left\{P_{3}^{1}, P_{3}^{2}\right\}\right)$ : 
$7 X_{1}+6 X_{2}-69=0$. Since $P_{3}^{0}$ belongs to the half-space, we have $E\left(\pi_{3}(T), P_{3}^{0}\right)$ :

$7 X_{1}+6 X_{2}-69 \leq 0$. By applying 3 , we obtain the discrete analytical description of the standard half-space $\mathbb{S} t\left(E\left(\pi_{3}(T), P_{3}^{0}\right)\right)=\left\{p \in \mathbb{Z}^{2} \mid 7 p_{1}+6 p_{2}<\frac{151}{2}\right\}$.

The half-space has a standard orientation (marked by a grey line in Figure $7)$. In the same way, we have $\mathbb{S} t\left(E\left(\pi_{3}(T), P_{3}^{1}\right)\right)=\left\{p \in \mathbb{Z}^{2} \mid-8 p_{1}+3 p_{2} \leq \frac{11}{2}\right\}$ with a supercover orientation and $\mathbb{S} t\left(E\left(\pi_{3}(T), P_{3}^{2}\right)\right)=\left\{p \in \mathbb{Z}^{2} \mid p_{1}-9 p_{2}<5\right\}$ with a standard orientation.

- The orthogonal projection $\pi_{1}\left(\pi_{3}(T)\right)$ is the interval $[0,8]$ and therefore $\mathbb{S} t\left(\varepsilon_{1}\left(\pi_{3}(T)\right)\right)=\left\{p \in \mathbb{Z}^{2} \mid \frac{-1}{2} \leq p_{2}<\frac{17}{2}\right\}$. The same way, we have $\mathbb{S} t\left(\varepsilon_{2}\left(\pi_{3}(T)\right)\right)=$ $\left\{p \in \mathbb{Z}^{2} \mid \frac{-1}{2} \leq p_{1}<\frac{19}{2}\right\}$.

- The discrete analytical description of the standard triangle is therefore:

$$
\mathbb{S} t\left(\pi_{3}(T)\right)=\left\{p \in \mathbb{Z}^{2} \mid \begin{array}{ccccccc}
7 p_{1} & +6 p_{2} & <\frac{151}{2} & ; & p_{2} & <\frac{17}{2} \\
-8 p_{1} & +3 p_{2} & \leq \frac{11}{2} & ; & -p_{2} & \leq \frac{1}{2} \\
p_{1} & -9 p_{2} & <5 & ; & p_{1} & <\frac{19}{2} \\
& & & & & & \\
& & & & & &
\end{array}\right.
$$

- The analytical descriptions of $\mathbb{S} t\left(\pi_{1}(T)\right)$ and $\mathbb{S} t\left(\pi_{2}(T)\right)$ are obtained in a similar way. The analytical description of the standard triangle $\mathbb{S} t(T)$ is defined by 17 discrete inequalities: 


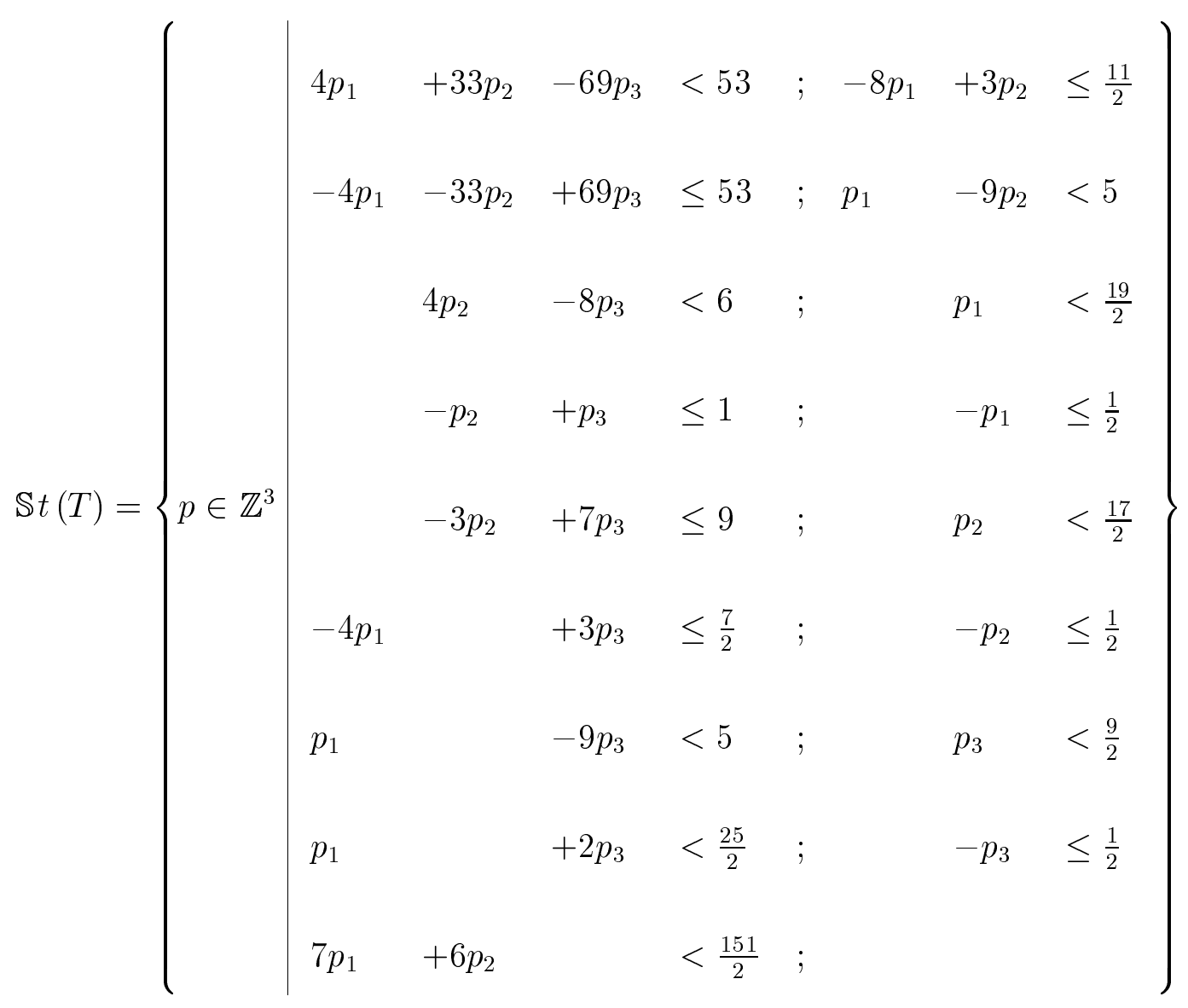

Figure 8 shows three views of the standard triangle $\mathbb{S} t(T)$ : Figure $8($ a) presents a classical, voxel view, of the standard triangle; figure 8(b) presents the same triangle in a cell-complex space representation; finally figure 8(c) represents what we have called the analytical view and represents the different inequalities describing the standard triangle.

\section{Conclusion}

We have defined in this paper the standard model for half-spaces, $m$-flats and simplices in dimension $n$. This is, to the authors best knowledge, the first time that a large class of geometrically consistent discrete primitives has been described analytically in dimension $n$. The standard model is geometrically 
consistent, defined analytically and standard objects are tunnel-free, $(n-1)$ connected and bubble-free.

The theoretical work in this paper needs now to be completed by the implementation of a standard object generation library for objects in dimension $n$. Such a library already exists as a prototype in dimension 2 and 3 . It is not very difficult to design linear generation algorithms (linear in the number of voxels belonging to the discrete object) in $2 \mathrm{D}$ and $3 \mathrm{D}$. The $3 \mathrm{D}$ standard line for instance can be generated by the algorithm proposed by Cohen-Or in 1997 [16]. It is more difficult however to design efficient generation algorithms in an arbitrary dimension. One major limitation of the standard model is that it is only defined for linear objects while the supercover is defined for arbitrary objects. The question of the extension of the standard model to arbitrary, non linear, objects remains an open question. The standard model is particularly well suited for the problem of the polygonalization. The border discrete 3D object can be decomposed into discrete polygons. Of course, in order to do this, a notion of discrete polygon was needed. The standard polygon seems to be an interesting answer as it comes with a definition of discrete 3D line and discrete $3 \mathrm{D}$ point. The actual algorithm that allows a discrete polygonalization and then transforming these discrete polygonals into Euclidean polygons is still a largely open question.

In order to facilitate the research on, the implementation and the test of such polygonalization algorithms, we are developing a discrete modeling tool, called SpaMod (for Spatial Modeler, see [8] for details), at the University of Poitiers (France). The standard model, as well as the supercover model, are part of the discrete objects models handled by Spamod. Spamod is still in the preliminary stages of its development, however Figure 8 and Figure 9 have been produced 


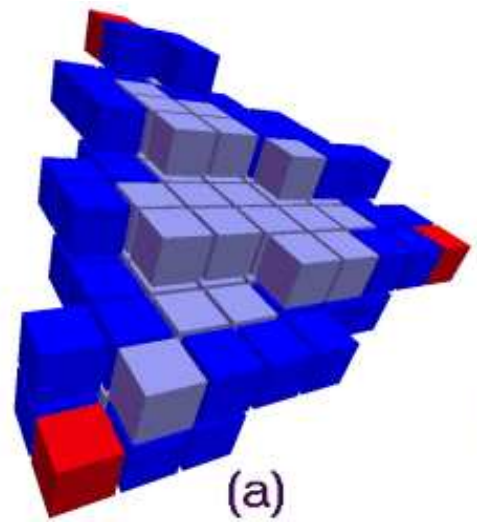

(a)

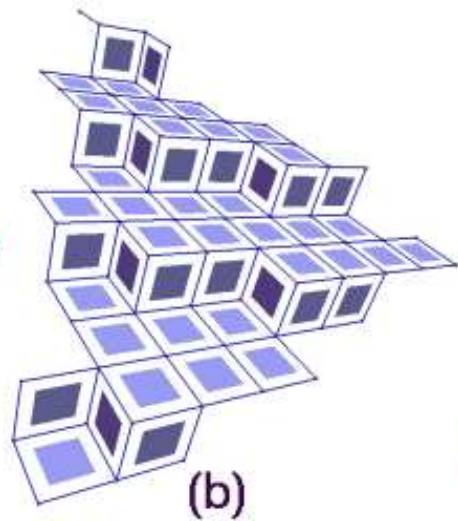

(b)

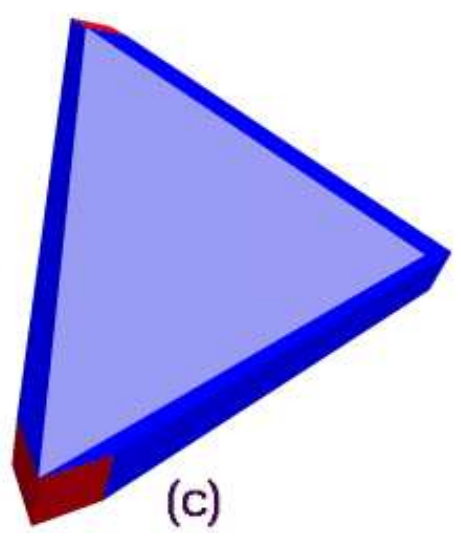

(c) Analytical view

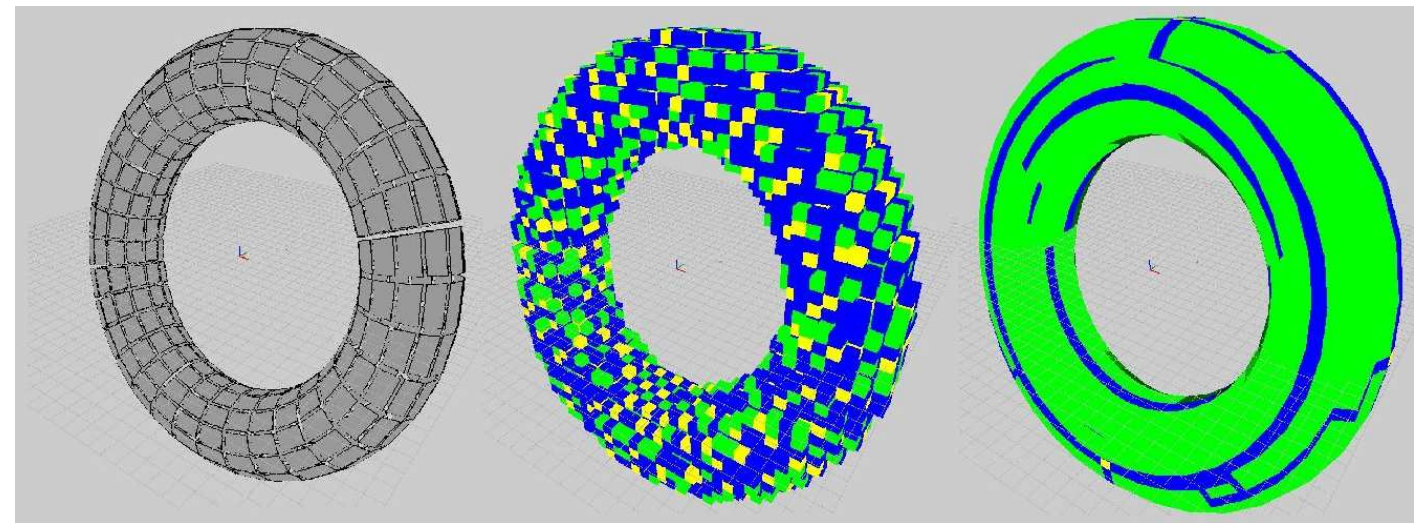

Fig. 9. From left to right, a Euclidean view of a polygonal torus, a voxel view and an analytical view.

with this software.

\section{References}

[1] J. Amanatides and A. Woo, A Fast Voxel Traversal Algorithm for Ray Tracing, in Proceedings, Eurographics '87, Amsterdam Netherlands (ed. G. Marechal ed.), pp. 3-9, Elsevier Science B.V., 1987.

[2] E. Andres, R. Acharya, C. Sibata, The Supercover 3D Polygon, 6th Int. Workshop on Discrete Geometry for Computer Imagery, Lyon France, Lecture 
Notes for Computer Science 1176, Springer Verlag, 1996, 237-242.

[3] E. Andres, M.-A. Jacob, The Discrete Analytical Hyperspheres, IEEE Transactions on Visualisation and Computer Graphics 3, $\mathrm{n}^{\circ} 1,1997,75-86$.

[4] E. Andres, R. Acharya, C. Sibata, Discrete Analytical Hyperplanes, Graphical Models and Image Processing 59, $\mathrm{n}^{\circ} 5$, 1997, 302-309.

[5] E. Andres, Ph. Nehlig, J. Francon, Tunnel-Free Supercover 3D Polygons and Polyhedra, in Proceedings Eurographics '97, Budapest Hungary, Computer Graphics Forum 16, n³, Blackwell Publishers, 1997, C3-C13.

[6] E. Andres, Ph. Nehlig and J. Françon, Supercover of Straight Lines, Planes and Triangles, $7^{\text {th }}$ Int. Workshop on Discrete Geometry for Computer Imagery, Montpellier France, Lecture Notes in Computer Science 1347, Springer Verlag, 1997, 243-253.

[7] E. Andres, Modélisation analytique discrète d'objets géométriques, Habilitation (in french), Laboratoire IRCOM-SIC, University of Poitiers France, 8 Dec. 2000.

[8] E. Andres, R. Breton, P. Lienhardt, SpaMod : design of a spatial modeling tool, Digital and Image Geometry, Advanced Lectures - Lecture Notes on Computer Science 2243, Springer Verlag, 2001, 91-107.

[9] E. Andres, Defining Discrete Objects for Polygonalization: the Standard Model, 10th Int. Workshop on Discrete Geometry for Computer Imagery, Bordeaux France, Lecture Notes for Computer Science 2301, Springer Verlag, 2002, 313325.

[10] J.E. Bresenham, Algorithms for computer control of a digital plotter, IBM Syst. Journal 4, $\mathrm{n}^{\circ} 1,1965,25-30$.

[11] V.E. Brimkov, E. Andres, R.P. Barneva, Object discretizations in high dimensions and membership recognition, $9^{\text {th }}$ Int. Workshop on Discrete 
Geometry for Computer Imagery, Uppsala Sweden, Lecture Notes in Computer Science 1953, Springer Verlag, 210-221.

[12] V.E. Brimkov, E. Andres, R.P. Barneva, Object discretizations in high dimensions, Pattern Recognition Letters 23, $\mathrm{n}^{\circ} 6,2002,623-636$.

[13] D. Cohen, Voxel traversal along a 3D line, in Graphics Gems IV (Paul Heckbert ed.), Academic Press, Boston USA, 1994.

[14] D. Cohen-Or, A.Kaufman, Fundamentals of surface voxelization, Graphical Models and Image Processing 57, $\mathrm{n}^{\circ} 6,1995,453-461$.

[15] D. Cohen-Or, A. Kaufman, T.Y. Kong, On the soundness of surface voxelization, in Topological algorithms for Image Processing (T.Y. Kong and A. Rosenfeld ed.), 1996, 181-204.

[16] D. Cohen-Or, A. Kaufman, 3D Line Voxelization and Connectivity Control, IEEE Computer Graphics and Applications, nov-dec. 1997, 80-87.

[17] O. Figueiredo, J.-P. Reveillès, A Contribution to 3D Digital Lines, in Proceedings $5^{\text {th }}$ Int. Workshop on Discrete Geometry for Computer Imagery, Clermont-Ferrand France, 1995, 187-198.

[18] J. Françon, Discrete Combinatorial Surfaces, Graphical Models and Image Processing 57, 1995.

[19] H. Heijmans, Morphological discretization, in Geometrical Problems in Image Processing (ed. U. Eckhardt et al.), Akademie Verlag, Berlin Germany, 1991, 99-106.

[20] G.T. Herman, Discrete Multidimensional Jordan Surfaces, Graphical Models and Image Processing 54, $\mathrm{n}^{\circ} 6,1992,507-515$.

[21] A. Kaufman, Efficient Algorithms for 3D Scan-Conversion of Parametric Curves, Surfaces, and Volumes, SIGGRAPH '87 Anaheim USA, Computer 
Graphics Forum 21, n 4 , 1987, 171-179.

[22] A. Kaufman, Efficient Algorithms for Scan-Converting 3D Polygons, Computer and Graphics 12, n 2, 1988, 213-219.

[23] A. Kaufman, D. Cohen, R. Yagel, Volume Graphics, IEEE Computer 26, $\mathrm{n}^{\circ} 7$, 1993, 51-64.

[24] C.E. Kim, Three-dimensional digital line segments, IEEE Transactions on Pattern Analysis and Machine Intelligence PAMI-5, 1983, 231-234.

[25] C.E. Kim, Three-dimensional digital planes, IEEE Transactions on Pattern Analysis and Machine Intelligence PAMI-6, n 5 , 1984, 639-645.

[26] C. Lincke, W. Wüthrich, Towards a unified approach between digitization of linear objects and discrete analytical objects, in Proceedings WSGG 2000 workshop (V.Skala ed.), University of West Bohemia, Plzen Tcheque Republic, 2000, 124-131.

[27] G.U. Montanari, On limit properties in digitization schemes, Journal of the $A C M 17, \mathrm{n}^{\circ} 2,1970,348-360$.

[28] A. Rosenfeld, T.Y. Kong, A.Y. Wu, Digital Surfaces, CGVIP 53, n4, 1991, 305-312.

[29] J. Serra, Image Analysis and Mathematical Morphology, Academic Press, London England, 1992.

[30] I. Stojmenovic, R. Tosic, Digitization schemes and the recognition of digital straight lines, hyperplanes and flats in arbitrary dimensions, Contemporay Math series, Vision Geometry 119, Amer. Math Soc. ed., Providence USA, 1991, 197212.

[31] M. Tajine, D. Wagner, C. Ronse, Hausdorff discretizations and its comparison to other discretization schemes, $8^{\text {th }}$ Int. Workshop on Discrete Geometry for 
Computer Imagery, Marne-la-Vallée France, Lecture Notes in Computer Science 1568, Springer Verlag, 1999, 399-410.

[32] P.Veelaert, On the flatness of digital hyperplanes, Journal of Mathematical Imaging and Vision 3, 1993, 205-221.

[33] D. Wagner, M. Tajine, C. Ronse, An approach to discretization based on Hausdorff metric, in Proceedings ISMM'98 (Kluwer ed.), Academic publisher, $1998,67-74$.

[34] C. Wüthrich, A model for curve rasterization in n-dimensional space, Computer 6 Graphics 22, n²-3, 1998, 153-160. 